\title{
Comparative Analysis of Color Video Enhancement Techniques
}

\author{
S.Rajeev ${ }^{1}$, P.Ravi Sankar ${ }^{2}$
}

${ }^{1}$ Department of ECE, Gudlavalleru Engineering College, Gudlavalleru, India

E-mail id:mtech3826@gmail.com

${ }^{2}$ Assistant Professor Department of ECE, Gudlavalleru Engineering College, Gudlavalleru, India E-mail id:RAVI482@gmail.com

\section{ABSTRACT}

Contrast enhancement has an important role in image processing applications. This paper presents a color enhancement algorithm based on adaptive filter technique. First, the proposed method is divided into three major parts: obtain luminance image and background image, adaptive adjustment and color restoration. Unlike traditional color image enhancement algorithms, the adaptive filter in the algorithm takes color information into consideration. The algorithm finds the importance of color information in color image enhancement and utilizes color space conversion to obtain a much better visibility. In the experimental results, the proposed method reproduces better enhancement and reduce the halo distortion compared with the bilateral methods.

\section{Indexing terms/Keywords}

Bilateral, Contrast enhancement, Halo distortion, Retinex and Adaptive adjustment.

\section{Council for Innovative Research}

Peer Review Research Publishing System

\section{Journal: International Journal of Computers \& Technology}

Vol 11, No.4

editor@cirworld.com

www.cirworld.com, member.cirworld.com 


\section{INTRODUCTION}

Recently, digital image processing techniques has been developed with the purpose of image enhancement, restoration, and so on. The field of digital color enhancement of old pictures, printings, and paintings are interested in the recent years. The old media generally lose their original colors with various reasons. Therefore, enhancement of faded image has been studied with various assumptions.

Color constancy methods are normally applied to correct the color of faded images, as the phenomenon of color fading is regarded as an illuminant effect. The most widely used color constancy methods are the gray world assumption and white patch Retinex methods [1]. The gray world assumption method estimates the illuminant using the average color of the pixels, whereas in the white patch Retinex method, if a bright patch in the image is a white patch in the scene, this patch then reflects the maximum light possible for each band. Meanwhile, a combination of these two methods has also been proposed, which uses a quadratic correction of two channels in order to simultaneously satisfy the gray world assumption and white patch Retinex [2].

Human eyes are very sensitivity and can find the light variation in faction of seconds. Human eyes have color adaptive ability to recognize high dynamic range. But the camera has high dynamic range and it is limited by its tone. It is usually occurs that video taken in so dark then details can't be seen easily. And videos taken by a camera is identical to an image sensed by human eyes. But these images are different in some situations due to the difference between human eyes and a digital camera [3-5]. The dynamic range of human eyes is four to six times as wide as a digital camera. Therefore, these differences especially in situations where strong luminance contrast exists. Hence, the real scene cannot be correlated to digital image. Most video enhancement methods attempt to analyze pixels using surrounding pixels to improve detail visibility [6]. To retain the video detail the bilateral tone adjustment is done in video frames. The idea of bilateral tone adjustment is to treat dark and bright regions separately using two different tones. Bilateral tone adjustment retrieve the details in dark and bright regions and but also boosts the contrast in the mid-tone regions .In this paper is organized as follows. Bilateral tone adjustment in section II. Section III describes the proposed method. Section IV describes the comparative performance. The simulation results are presented in Section V. Concluding remarks are made in Section VI.

\section{BILATERAL TONE ADJUSTMENT TECHNIQUE (BTAT)}

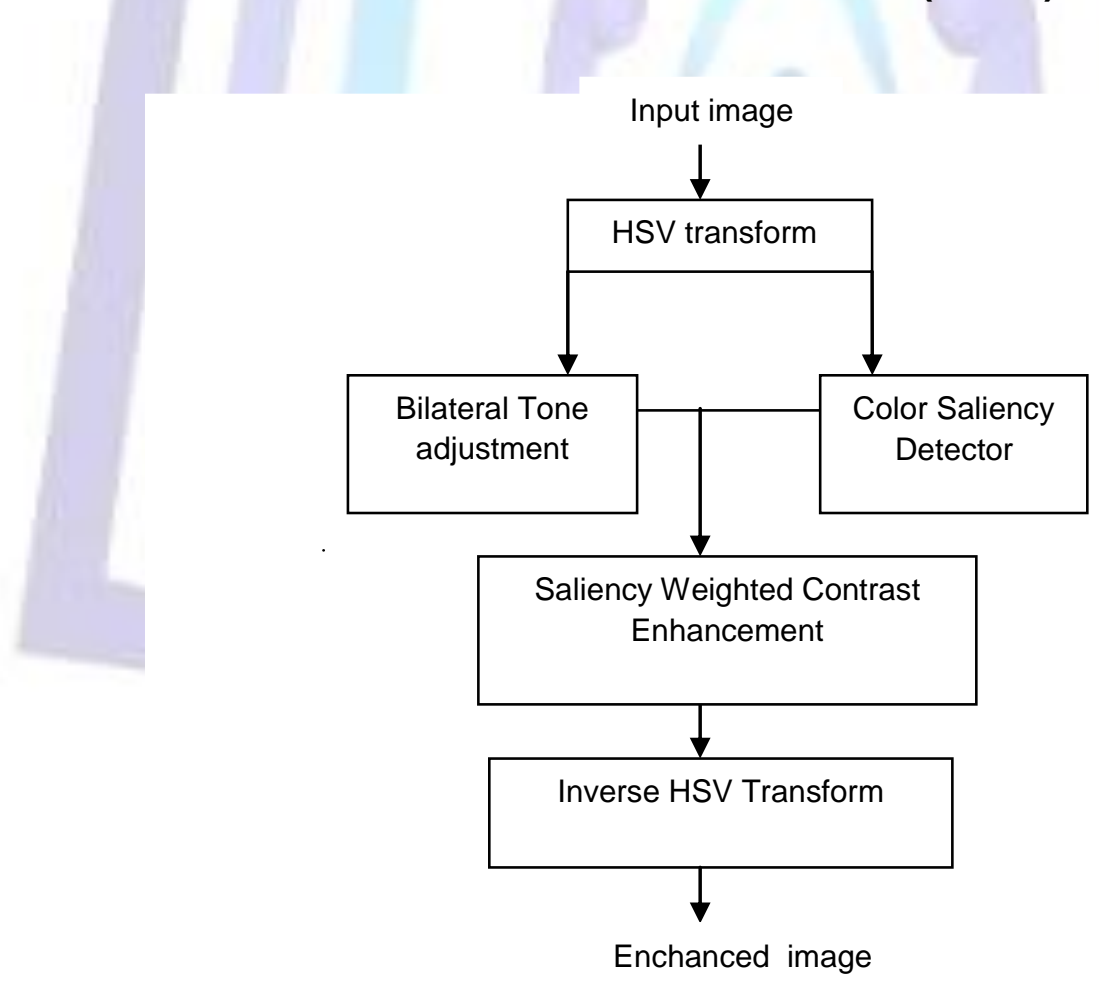

Figure 1: Flow diagram for BTAT.

The following steps are used to improve the color enhancement using bilateral tone adjustment. First step is to adjust brightness RGB cannot be used because each of the three color channels would get changed and changing them by the same amount to adjust brightness will usually shift the color. So the input RGB image is transformed into the HSV domain to derive the luminance and color maps.

The second step is the bilateral gamma adjustment (BiGA) is used to adjust the global luminance using the gamma adjustment function by taking value as its input. Global luminance is to reassign the intensity values of pixels to make the intensity distribution uniform. This method improves the visibility of details in dark and bright region. 
$\mathrm{Ga}$ is the gamma adjustment factor which

$$
\mathrm{Ga}(\mathrm{L})=(\mathrm{Gd}+\mathrm{Gb}) / 2
$$

function for enhancing dark and bright region.

enhance the contrast in bright and dark region. $\mathrm{Gd}$ and $\mathrm{Gb}$ are the

The third step is color saliency detector. This detector produces the saliency map by taking hue and saturation as input with well defined boundary of salient object. The saliency map S for an image I with width $\mathrm{W}$ and a height $\mathrm{H}$ is given as follows,

$$
\mathrm{S}(\mathrm{x}, \mathrm{y})=[\mathrm{l}-\operatorname{lwh} c(\mathrm{x}, \mathrm{y})]
$$

Where $\mathrm{l}=$ arithmetic mean pixel value of the image

$\operatorname{lwhc}(x, y)=$ image pixel vector value of original image

The final step is Saliency Weighted Contrast Enhancement. This technique produces high contrast in region with higher extent to human interest. In addition, the noise is not over enhanced because SWCE controls the enhancement extent adaptively depending on local saliency values.

A simple approach to enhance contrast is shown below,

$$
L=L b+\beta s a l(L-m)
$$

\section{PROPSED METHOD}

The algorithm proposed consists of three major parts: (1) obtain luminance image and background image, adaptive adjustment, (3) color restoration as shown in Figure 2.

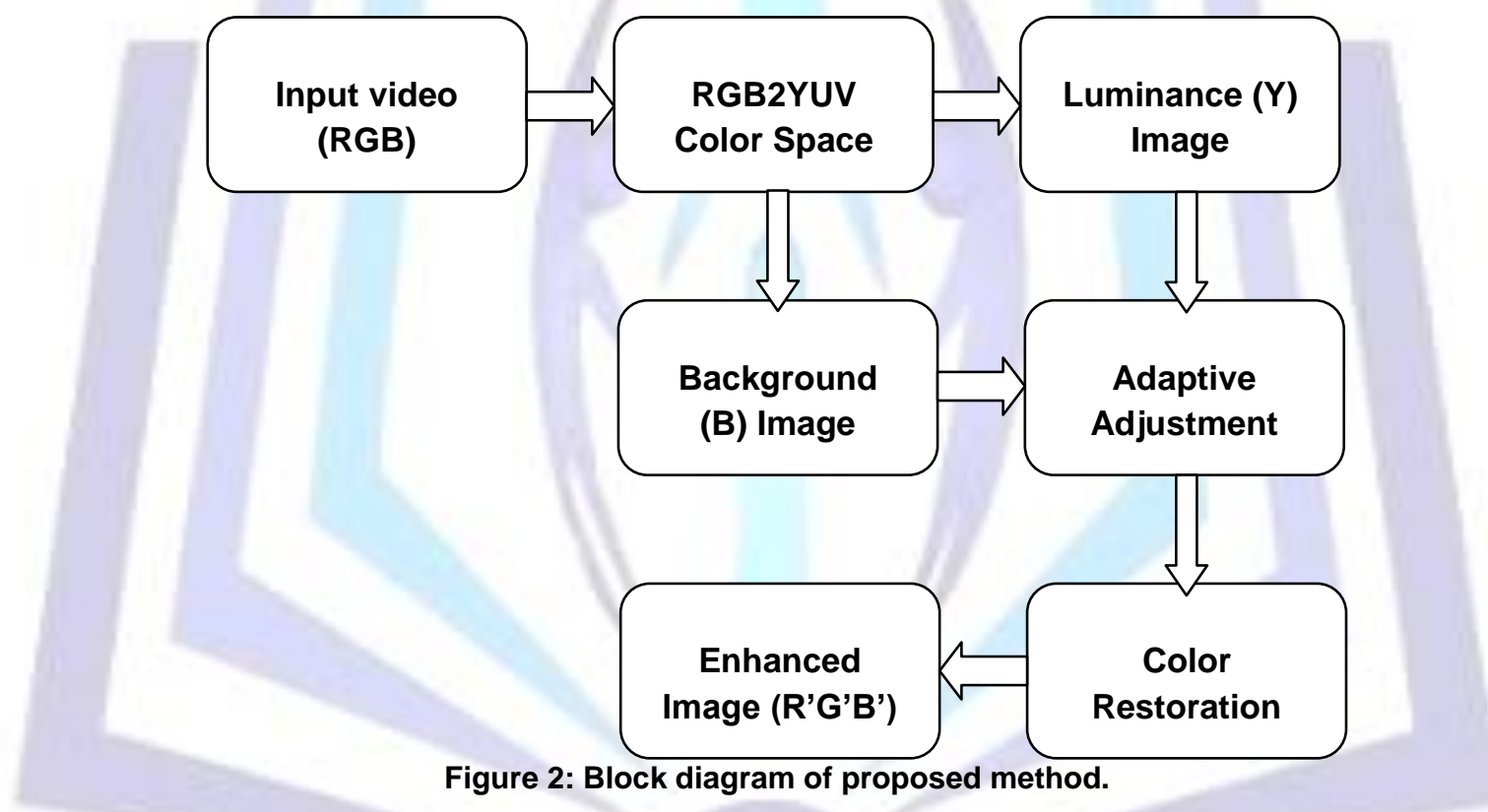

The first step is the color images we usually see are mostly in RGB color space, which employ red, green, and blue three primary colors to produce other colors. In RGB color space, other colors are synthesized by three primary colors, which is not effective in some cases. Consequently, we use another color space-YUV color space instead of the RGB color space in the algorithm proposed. The importance of using YUV color space is that its brightness image $Y$ and chroma images $\mathrm{U}, \mathrm{V}$ are separate. $\mathrm{Y}$ stands for the luminance, and $\mathrm{U}, \mathrm{V}$ are color components. After that to obtain the background image according to the $\mathrm{Y}, \mathrm{U}, \mathrm{V}$ values at pixel $(\mathrm{x}, \mathrm{y})$.

Where

$$
I_{B}(x, y)=\frac{\sum G_{R} G_{N} N(x, y)}{\sum G_{R} G_{N}}
$$

The $N(x, y)$ represents the pixel of $(x, y)$.

$\mathrm{G}_{N}$ is the scale parameter of pixel filtering

$G_{R}$ is the distance parameter of intensity image.

We use the below formula to obtain the distance parameter

$$
G_{R}\left(x, y, x_{i}, y_{j}\right)=\exp \left\{-\frac{\left(x-x_{i}\right)^{2}+\left(y-y_{j}\right)^{2}}{2 \sigma_{R}^{2}}\right\}
$$


Here (xi,yi) is the neighbor pixels of $\mathrm{Y}, \mathrm{U}, \mathrm{V}$ values.

$\mathrm{G}_{\text {| }}$ is the distance parameter of $\mathrm{U}, \mathrm{V}$ image. We use the below formula to obtain the distance parameter

$$
\left.G_{I}\left(x, y ; x_{i}, y_{j}\right)=\exp -\frac{\left(I(x, y)-I\left(x_{i}, y_{j}\right)\right.}{2 \sigma_{I}^{2}}\right\}
$$

$\mathrm{G}_{\mathrm{c}}$ is the scale parameter of pixel filtering

$$
\left.G_{c}\left(x, y, x_{i}, y_{j}\right)=\exp -\frac{\left(U(x, y)-U\left(x_{i}, y_{j}\right)\right)^{2}+\left(V(x, y)-V\left(x, y_{j}\right)^{2}\right.}{2 \sigma_{C}^{2}}\right\}
$$

Here $, U(x, y), V(x, y)=$ chrome images of $Y U V$ image

$I(x, y)=$ intensity value at $(x, y)$

$\sigma R, \sigma l, \sigma C$ are the scale parameters, whose values are 20,30,60 respectively.

The second step is adaptive adjustment. By using adaptive adjustment to obtain the local enhancement IE(x,y).

We use the formula to obtain the local enhancement

$$
\operatorname{IE}(x, y)=\beta(x, y) \cdot I L(x, y) \text {; }
$$

$\beta(x, y)$ is the function of adaptive regulation. $I E(x, y)$ is local enhanced color image, and the enhanced color image can be obtained after the color restoration for IE.

$$
\text { Where } \beta(x, y)=(a \alpha+b) \cdot w(x, y) \text {; }
$$

where, $\alpha$ is intensity coefficient according to the cumulative distribution function (CDF) of the luminance image. $\mathrm{w}(\mathrm{x}, \mathrm{y})$ is the ratio value between the background image and the intensity image. $a$ and $b$ are constants, wecan adjust them to achieve good adjustment results.

The third step is color restoration. To apply the fast Fourier transform (FFT) of IE we can get the image I'.

$$
\begin{aligned}
& R^{\prime}(x, y)=R(x, y) I^{\prime}(x, y) / I(x, y) \\
& G^{\prime}(x, y)=G(x, y) I^{\prime}(x, y) / I(x, y) \\
& B^{\prime}(x, y)=B(x, y) l^{\prime}(x, y) / I(x, y)
\end{aligned}
$$

The enhanced images' qualities usually evaluated through subjective measurement. For the objective estimation, global mean and contrast enhancement index are used to assess enhanced images. The software coding for the hardware functionality is written in embedded $C$ language in Keil software.

\section{IMAGE QUALITY MEASUREMENTS}

There are different quantitative measures which are used to evaluate the performance of the fusion techniques. We used three measures Root Mean Square Error (RMSE), Peak Signal to Noise Ration (PSNR), Mutual Information (MI), entropy and standard deviation.

The RMSE of reference image $R$ and fused images $F$ is given by

$$
R M S E=\frac{1}{M * N} \sum_{i=1}^{m} \sum_{j=1}^{n}[R(i, j)-F(i, j)]^{2}
$$

The PSNR of reference image $R$ and fused images $F$ is given by

$$
P S N R=20 \log 10 \frac{\mathrm{L}^{2}}{\frac{1}{M * N} \sum_{i=1}^{m} \sum_{j=1}^{n}[R(i, j)-F(i, j)]^{2}}
$$

Where $L$ is the number of gray levels

The PSNR of reference image $R$ and fused images $F$ is given by

$$
M I=\frac{1}{M^{*} N} \sum_{i=1}^{m} \sum_{j=1}^{n}[h(i, j)] \log 2\left[\frac{h(i, j)}{h(i, j) T(i, j)}\right]
$$

Where $\mathrm{h}$ is the fused image 


\section{RESULTS}

In this paper, the adaptive filter referred proposes a solution in reducing halo and achieves a better visibility compare to bilateral tone adjustment and comparative analysis of both techniques.

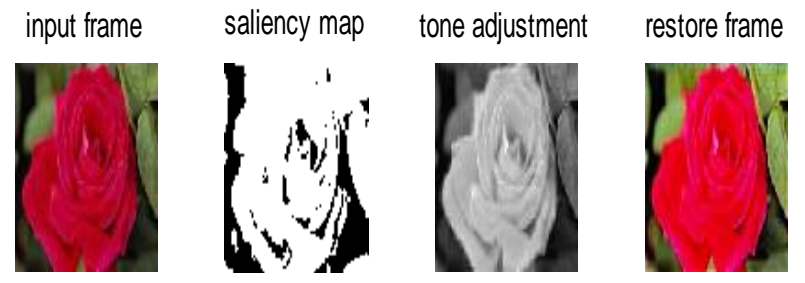

Figure 3: Enhanced results of using the BTAT algorithm.

orginal frame

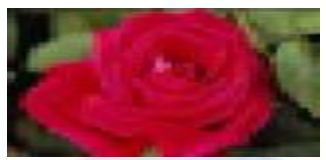

Restore image

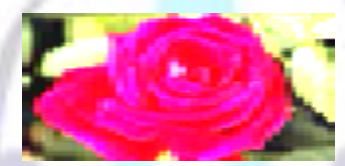

Background image

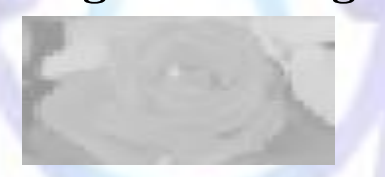

Figure 4: Enhanced results of using the proposed algorithm.

Table 1 lists the objective evaluation for the enhanced color images in the Figure 3 and 4.

Table.1 Comparison table for Parameter Calculation

\section{CONCLUSION}

\begin{tabular}{|l|l|l|}
\hline \multicolumn{1}{|c|}{ TECHNIQUES } & \multicolumn{1}{|c|}{ MSE } & PSNR \\
\hline BTAT & 0.98 & 25.43 \\
\hline PROPOSED & 0.5631 & 35.26 \\
\hline
\end{tabular}

A new color video enhancement algorithm is proposed in this paper. In adaptive filter technique, MSE value and mean brightness error values are reduced simultaneously comparing to BTAT algorithm. Adaptive filter technique maintains its power reduction obtained from BTAT algorithm with improved image quality than BTAT Algorithm. Hence adaptive filter technique produces better contrast enhancement results comparing to BTAT Algorithm.

\section{REFERENCES}

[1] Wei-MingKe, Chih-RungChen,andChing-TeChiu "Image Enhancement with Bilateral Tone Adjustment and Saliency Weighted ContrastEnhancement" IEEETRANSACTIONS ON CIRCUITS AND SYSTEMS FOR VIDEO TECHNOLOGY ,VOL.21, NO.3 ,MARCH2011

[2] Y.-T. Kim, "Contrast enhancement using brightness preserving bihistogram equalization," IEEE Trans. Consumer Electron., vol. 43, no. 1, pp. 1-8, Feb. 1997.

[3] S.-D. Chen and A. R. Ramli, "Minimum mean brightness error bihistogram equalization in contrast enhancement," IEEE Trans. Consumer Electron., vol. 49, no. 4, pp. 1310-1319, Nov. 2003.

[4] C. Wang and Z. Ye, "Brightness preserving histogram equalization with maximum entropy: A variational perspective," IEEE Trans. Consumer Electron., vol. 51, no. 4, pp. 1326-1334, Nov. 2005.

[5] T.-K. Kim, J.-K. Paik, and B.-S. Kang, "Contrast enhancement system spatially adaptive histogram equalization with temporal filtering," IEEE Trans. Consumer Electron., vol. 44, no. 1, pp. 82-87, Feb. 1998. 
[6] J.-Y. Kim, L.-S. Kim, and S.-H. Hwang, "An advanced contrast enhancement using partially overlapped sub-block histogram equalization," IEEE Trans. Circuit Syst. Video Technol., vol. 11, no. 4, pp. 475-484, Apr. 2001. S. Cvetkovic, J. Schirris, and P. de With, "Locally -adaptive image contrast enhancement without noise and ringing artifacts," in Proc. ICIP, vol. 3. 2007, pp. 551-560.

[7] D. Chang and W. Wu, "Image contrast enhancement based on a histogram transformation of local standard deviation," IEEE Trans. Med. Imaging, vol. 17, no. 4, pp. 518-531, Apr. 1998.

[8] C. Siagian and L. Itti, "Rapid biologically -inspired scene classification using features shared with visual attention," IEEE Trans. Pattern Anal.Mach. Intell., vol. 29, no. 2, pp. 300-312, Feb. 2007.

[9] L. Itti, "Automatic foveation for video compression using a neurobiological model of visual attention," IEEE Trans. Image Process., vol. 13, no. 10, pp. 1304-1318, Oct. 2004.

\section{Author' biography with Photo}

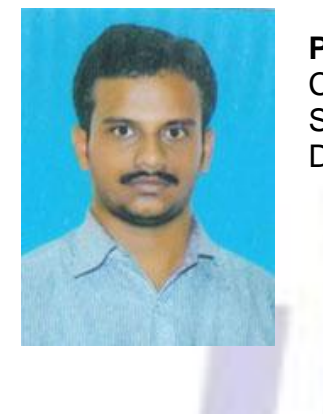

P.Ravisankar obtained his Bachelor's Degree \& Master's degree from Gudlavalleru Engineering College, Gudlavalleru.his Areas of interest are Microprocessors, Microcontrollers, Embedded System De sign, Low Power VLSI Design.Presently working as Assistant Professor ECE Department,GudlavalleruEngineering College. Gudlavalleru, India

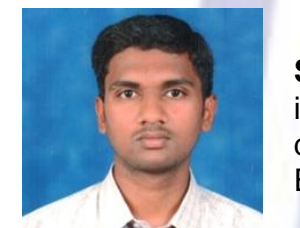

S.Rajeev obtained his Bachelor's Degree from Narayana college of Engineering, Nellore. His Areas of interest in Digital image processing, Embedded System Design, Low Power VLSI Design. Presently is doing M.Tech digital Electronics and communication systems at Dept.of ECE, Gudlavalleru Engineering College, Gudlavalleru, India. 